\title{
Fibrinogen is associated with glucose metabolism and cardiovascular outcomes in patients with coronary artery disease
}

Shuo-Lin Liu, Na-Qiong Wu', Hui-Wei Shi, Qian Dong, Qiu-ting Dong, Ying Gao, Yuan-Lin Guo and Jian-Jun Li

\begin{abstract}
Background: The present cohort study aims to examine the relationship between fibrinogen (Fib) levels and glucose metabolism [fasting blood glucose (FBG) and hemoglobin $\mathrm{A} 1 \mathrm{c}(\mathrm{HbA} 1 \mathrm{c})$ ] and investigate the impact of high Fib on cardiovascular outcomes in patients with stable CAD and pre-diabetes mellitus (pre-DM) or diabetes mellitus (DM).

Methods: This study included 5237 patients from March 2011 to December 2015. Patients were distributed into three groups according to Fib levels (low Fib, median Fib, high Fib) and further categorized by glucose metabolism status [normal glucose regulation (NGR), Pre-DM, DM]. All patients were followed up for the occurrences of major adverse cardiovascular events (MACEs), including cardiovascular mortality, nonfatal MI, stroke, and unplanned coronary revascularization.

Results: Linear regression analyses showed that FBG and $\mathrm{HbA} 1 \mathrm{c}$ levels were positively associated with Fib in overall CAD participants, either with or without DM (all $P<0.001$ ). During an average of 18,820 patient-years of follow-up, 476 MACEs occurred. High Fib was independently associated with MACEs after adjusting for confounding factors [Hazard Ratio (HR): 1.57, 95\% confidence interval (Cl) 1.26-1.97, $P<0.001$ ]. Furthermore, DM but not pre-DM was a significant predictor of MACEs $(P<0.001$ and $P>0.05$, respectively). When patients were stratified by both glucose metabolism status and Fib levels, high Fib was associated with a higher risk of MACEs in pre-DM (HR 1.66, 95\% Cl 1.02-2.71, $P<0.05)$. Medium and high Fib levels were associated with an even higher risk of MACEs in DM (HR $1.86,95 \% \mathrm{Cl}$ 1.14-3.05 and HR 2.28, 95\% Cl 1.42-3.66, all $P<0.05$ ). After adding the combination of Fib and glucose status to the Cox model, the C-statistic was increased by 0.015 (0.001-0.026).

Conclusions: The present study suggested that Fib levels were associated with FBG and $\mathrm{HbA} 1 \mathrm{c}$ in stable CAD patients. Moreover, elevated Fib was independently associated with MACEs in CAD patients, especially among those with pre-DM and DM, suggesting that Fib may provide incremental value in the cardiovascular risk stratification of pre-DM and DM patients.
\end{abstract}

Keywords: Fibrinogen, HbA1c, Fasting blood glucose, Diabetes mellitus, Coronary artery disease, Prognosis

*Correspondence: fuwainaqiongwu@163.com

Endocrinology \& Cardiometabolic Center, State Key Laboratory of Cardiovascular Disease, Fuwai Hospital, National Center

for Cardiovascular Diseases, Chinese Academy of Medical Sciences and Peking Union Medical College, No 167 BeiLiShi Road, XiCheng District, Beijing 100037, China

\section{Background}

Fibrinogen (Fib), as a thromboplastic and inflammatory marker, facilitates blood viscosity, platelet aggregation, fibrin cross-linking, and plays a pivotal role in the progression of atherosclerosis [1, 2]. Previous studies showed that Fib was not only an indicator of subclinical cardiovascular diseases, such as coronary artery calcification and intima-media thickness of the carotid artery [3,

(c) The Author(s) 2020. This article is licensed under a Creative Commons Attribution 4.0 International License, which permits use, sharing, adaptation, distribution and reproduction in any medium or format, as long as you give appropriate credit to the original author(s) and the source, provide a link to the Creative Commons licence, and indicate if changes were made. The images or other third party material in this article are included in the article's Creative Commons licence, unless indicated otherwise in a credit line to the material. If material is not included in the article's Creative Commons licence and your intended use is not permitted by statutory regulation or exceeds the permitted use, you will need to obtain permission directly from the copyright holder. To view a copy of this licence, visit http://creativeco mmons.org/licenses/by/4.0/. The Creative Commons Public Domain Dedication waiver (http://creativecommons.org/publicdomain/ zero/1.0/) applies to the data made available in this article, unless otherwise stated in a credit line to the data. 
4], but was also independently associated with the development of coronary artery disease (CAD), hypertension, stroke, as well as adverse cardiovascular events [5-9].

The prevalence of type 2 diabetes mellitus (DM) and its cardiovascular complications has increased significantly worldwide [10]. In China, the prevalence of DM and prediabetes mellitus (pre-DM) were also steadily increasing, with a rate of DM in adults reaching $10.9 \%$ and pre-DM reaching $35.7 \%$ in 2013 [11]. Chronic, low-grade inflammation is an important predisposing factor for DM, and also contributes to the genesis of diabetes complications [12]. Fib, one of the subclinical inflammation biomarkers, was reported to increase before the onset of DM [13], and elevate from normal glucose regulation (NGR) over pre-DM to DM [14]. In the meanwhile, it has been demonstrated that Fib level was significantly associated with glucose metabolism [including fasting blood glucose (FBG) and glycosylated hemoglobinA1c (HbA1c), a measure of long-term glycemic control] in patients with acute coronary syndromes (ACS) [15]. Nevertheless, few reports have explored the relationship between serum Fib levels and glucose metabolism in patients with newonset stable CAD. Moreover, Fib was also implicated in the presence of macrovascular complications and microvascular disorders in DM, [14, 16, 17] while there has been no study to investigate the impact of Fib levels on cardiovascular risk in individuals with impaired glucose regulation.

The present cohort study was conducted to examine the relationship between serum Fib levels and glucose metabolism, and investigate the combined effect of high Fib and pre-DM on cardiovascular outcomes in a large cohort of patients with stable CAD.

\section{Materials and methods}

\section{Study design and population}

The present study was designed as a single-center, observational cohort study. As described in the flowchart (Fig. 1), from March 2011 to December 2015, 8022 patients who had received coronary angiography examination because of angina-like chest pain or positive noninvasive tests (such as treadmill exercise test or coronary computed tomography angiography) were recruited at the Fuwai Hospital, Chinese Academy of Medical Sciences. Then, 303 patients with the detailed data lost and 887 patients without angiographically determined CAD were excluded. Moreover, 1309 patients with acute coronary syndrome (ACS), previous myocardial infarction (MI), previous percutaneous coronary artery intervention (PCI) or coronary artery bypass grafting (CABG), 258 Patients with congestive heart failure, systematic inflammatory disease,

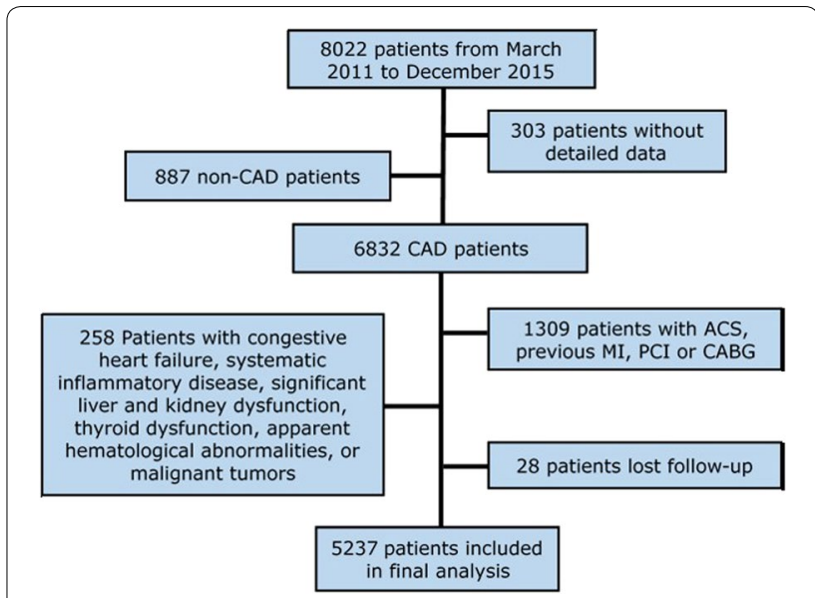

Fig. 1 Flowchart of the study population. ACS acute coronary syndrome, $C A D$ coronary artery disease, $P C /$ percutaneous coronary intervention, $C A B G$ coronary artery bypass grafting

significant liver and kidney dysfunction, thyroid dysfunction, apparent hematological abnormalities, or malignant tumors, and 28 patients lost to follow-up were excluded. Ultimately, 5237 patients were included in the final analysis. The patients were followed up regularly, as outlined in the study protocol. The study complied with the principles of the Declaration of Helsinki and was approved by the ethical review board of Fuwai Hospital (Beijing, China). Written informed consent was obtained from all participants.

All patients were followed up semiannually through telephone interviews or clinic visits. Trained clinical physicians or nurses who were blinded to previous medical histories accomplished the interview. All clinical events were carefully examined by three independent cardiologists. The major adverse cardiovascular events (MACEs) were cardiovascular mortality, nonfatal MI, stroke (hemorrhagic stroke or ischemic stroke), and unplanned coronary revascularization (PCI and CABG). Deaths of participants were informed by relatives, medical records, or physicians. The composite endpoints included cardiovascular mortality, nonfatal MI, and nonfatal stroke [18].

According to the American Diabetes Association criteria [19], DM was confirmed by a fasting blood glucose (FBG) level $\geq 7.0 \mathrm{mmol} / \mathrm{L}$, or 2 -h blood glucose level $\geq 11.1 \mathrm{mmol} / \mathrm{L}$, or $\mathrm{HbA} 1 \mathrm{c}$ level $\geq 6.5 \%$, or currently using hypoglycemic medications. Pre-DM was defined as any nondiabetic patients who had an FBG ranges from 5.6 to $<7.0 \mathrm{mmol} / \mathrm{L}$, or 2-h glucose ranges from 7.8 to $<11.1 \mathrm{mmol} / \mathrm{L}$, or $\mathrm{HbA} 1 \mathrm{c}$ level ranges from 5.7 to $<6.5 \%$. NGR represented participants without pre-DM or DM. 


\section{Laboratory tests}

Blood samples were taken from patients in a fasting state for at least 12 -h in the morning. The enzymatic hexokinase method was used to determine glucose concentrations. HbA1c was evaluated by Tosoh Automated Glycohemoglobin Analyser (HLC-723G8, Tokyo, Japan). The Fib levels were measured by a Stago autoanalyser with the STA Fibrinogen kit (Diagnostic Stago, 101 Taverny, France). All other laboratory parameters were analyzed at the biochemistry center of our hospital by standard biochemical tests.

\section{Statistical analysis}

The statistical analyses were performed with SPSS version 22.0 software (SPSS Inc., Chicago, IL, USA) and R language version 3.5.2 (Eggshell Igloo). Missing values were dealt with multiple imputation method [20]. Continuous variables were presented as mean \pm standard deviation (SD) or median (interquartile range). Categorical variables were presented as number (percentage). The distributions of parameters were examined by the Kolmogorov-Smirnov test. $P$ values for trend across Fib levels in the continuous variables were tested by a generalized linear model. The post hoc multiple comparisons among groups were analyzed by Student's $t$-test, one-way ANOVA or Mann-Whitney $\mathrm{U}$ test where appropriate. $\mathrm{P}$ values for trend in the categorical variables were compared by Chi-square test or Fisher exact test. Linear regression analyses were performed to measure the independent relationships between $\mathrm{HbA1c}$ and Fib or FBG and Fib. The event-free survival rates among groups were determined by the Kaplan-Meier method and compared by the log-rank test. Univariate and multivariate Cox proportional hazard regressions were used to calculate the hazard ratio (HR) and 95\% confidence interval (CI). The adjusted Cox models were adjusted for risk factors as follows: age, sex, body mass index (BMI), smoking, hypertension, family history of coronary artery disease, left ventricular ejection fraction (LVEF), low density lipoprotein cholesterol (LDL-C), high lipoprotein cholesterol (HDL-C), Lntransformed triglyceride (TG), Ln-transformed highsensitivity C-reactive protein ( $\mathrm{HsCRP})$, and creatinine. The proportional hazard assumption for each Cox regression models was tested using Schoenfeld residuals and checked with smoothed plots of Schoenfeld residuals (all variables satisfy the $\mathrm{PH}$ assumption of $\mathrm{Cox}$ models). $\mathrm{C}$-statistic and $\Delta \mathrm{C}$-statistic were calculated to evaluate the efficiency of models and the incremental value of adding the combination of Fib and glucose metabolism status into the original model. A value of $P<0.05$ was considered statistically significant.

\section{Results}

Baseline characteristics

Patients were distributed into three groups, according to Fib levels. The baseline characteristics of the study population are shown in Table 1. Patients with higher Fib levels were more likely to be the female and the elderly, had higher BMI, FBG and HbA1c levels, higher lipid levels (including TC, LDL-C, and TG) and HsCRP levels, but lower LVEF (all $P<0.05$ ) than those with low Fib levels. Moreover, the percentages of hypertension and DM were higher (all $P<0.001$ ) in patients with higher Fib levels, while the percentages of current smokers and drinkers were lower (all $P=0.002$ ) with the increase of Fib level. There were also significant differences in medications at discharge among the three groups. However, no significant differences were observed regarding the family history of CAD, HDL-C, and creatinine among the participants (all $p>0.05$ ).

\section{Association of glucose metabolism with Fib}

Linear regression analyses were performed to explore the association between glucose metabolism indexes (FBG and HbA1c) and Fib (Table 2). HbA1c level $\left(R^{2}=0.027\right.$; Standard $\beta=0.166, P<0.001)$ and FBG $\left(R^{2}=0.014\right.$; Standard $\beta=0.120, P<0.001$ ) were positively associated with Fib in overall participants with CAD. Furthermore, in CAD patients with DM, HbA1c level $\left(R^{2}=0.045\right.$; Standard $\beta=0.213, P<0.001)$ and FBG level $\left(R^{2}=0.021\right.$; Standard $\beta=0.146, \quad P<0.001)$ were also significantly positively associated with Fib. Additionally, linear regression analysis indicated positive associations of Fib with HbA1c $\left(R^{2}=0.018\right.$; Standard $\left.\beta=0.135, P<0.001\right)$ and FBG $\left(R^{2}=0.004\right.$; Standard $\left.\beta=0.065, P<0.001\right)$ in nonDM population (Fig. 2).

\section{Fib levels and cardiovascular outcomes}

Over an average of 18,820 patient-years of follow-up, 476 MACEs occurred (52 experienced cardiac death, 62 suffered nonfatal MI, 131 had strokes, and 231 received unplanned revascularization). The corresponding prevalence of MACEs in the low Fib, medium Fib, and high Fib group was $7.2 \%, 9.2 \%$, and $10.9 \%$, respectively. Univariate Cox proportional hazard regression analyses revealed that per SD change of Fib (HR: 1.18, 95\% CI 1.09-1.27, $P<0.001$ ), medium Fib (HR: 1.30, 95\% CI 1.03-1.64, $P=0.029$ ), high Fib (HR: $1.57,95 \%$ CI $1.26-1.97, P<0.001)$ were significantly associated with increased MACEs. After adjusting for potential confounders including age, sex, BMI, smoking, hypertension, family history of CAD, LVEF, LDL-C, HDL-C, Ln-transformed TG, Ln-transformed HsCRP, and creatinine, the multivariate cox proportional hazard regression analysis showed that Fib was also independently associated 
Table 1 Baseline characteristics of study population according to fibrinogen levels

\begin{tabular}{|c|c|c|c|c|c|}
\hline & $\begin{array}{l}\text { Total } \\
(n=5237)\end{array}$ & $\begin{array}{l}\text { Low Fib } \\
(<2.82 \mathrm{mg} / \mathrm{dL}) \\
(\mathrm{n}=1746)\end{array}$ & $\begin{array}{l}\text { Medium Fib } \\
(2.82-3.39 \mathrm{mg} / \mathrm{dL}) \\
(\mathrm{n}=1746)\end{array}$ & $\begin{array}{l}\text { High Fib } \\
(>3.39 \mathrm{mg} / \mathrm{dL}) \\
(\mathrm{n}=1745)\end{array}$ & $\mathrm{p}$-value for trend \\
\hline Age, years & $57.79 \pm 10.11$ & $56.23 \pm 9.91$ & $57.86 \pm 9.97$ & $59.28 \pm 10.21$ & $<0.001$ \\
\hline Male, n (\%) & $3729(71.2)$ & $1372(78.6)$ & $1241(71.1)$ & $1113(63.8)$ & $<0.001$ \\
\hline $\mathrm{BMI}\left(\mathrm{kg} / \mathrm{m}^{2}\right)$ & $25.78 \pm 3.17$ & $25.65 \pm 3.08$ & $25.76 \pm 3.02$ & $25.92 \pm 3.39$ & 0.041 \\
\hline Hypertension, n (\%) & $3320(63.4)$ & $1047(60.0)$ & $1114(63.8)$ & $1158(66.4)$ & $<0.001$ \\
\hline Diabetes, n (\%) & $1637(31.3)$ & $445(25.5)$ & $567(32.5)$ & $625(35.8)$ & $<0.001$ \\
\hline Family history of CAD, $n(\%)$ & $812(15.5)$ & $276(15.8)$ & $267(15.3)$ & $267(15.3)$ & 0.916 \\
\hline Current Smokers, n (\%) & $2807(53.6)$ & $981(56.2)$ & $946(54.2)$ & $879(50.4)$ & 0.002 \\
\hline Drinkers, n (\%) & $1628(31.1)$ & $597(34.2)$ & $529(30.3)$ & $502(28.8)$ & 0.002 \\
\hline $\mathrm{FBG}(\mathrm{mmol} / \mathrm{L})$ & $5.75 \pm 1.72$ & $5.52 \pm 1.53$ & $5.74 \pm 1.68$ & $5.98 \pm 1.90$ & $<0.001$ \\
\hline $\mathrm{HbA1c}(\%)$ & $6.33 \pm 1.10$ & $6.09 \pm 0.92$ & $6.35 \pm 1.09$ & $6.54 \pm 1.23$ & $<0.001$ \\
\hline Creatinine ( $\mu$ mol) & $76.61 \pm 18.68$ & $15.92 \pm 0.38$ & $17.64 \pm 0.42$ & $21.96 \pm 0.53$ & 0.297 \\
\hline $\mathrm{TC}(\mathrm{mmol} / \mathrm{L})$ & $4.17 \pm 1.16$ & $4.00 \pm 1.07$ & $4.19 \pm 1.14$ & $4.31 \pm 1.24$ & $<0.001$ \\
\hline $\mathrm{HDL}-\mathrm{C}(\mathrm{mmol} / \mathrm{L})$ & $1.05 \pm 0.28$ & $1.05 \pm 0.28$ & $1.04 \pm 0.27$ & $1.06 \pm 0.30$ & 0.463 \\
\hline LDL-C (mmol/L) & $2.54 \pm 1.00$ & $2.41 \pm 0.95$ & $2.54 \pm 0.95$ & $2.66 \pm 1.07$ & $<0.001$ \\
\hline $\mathrm{TG}(\mathrm{mmol} / \mathrm{L})$ & $1.52(1.13-2.12)$ & $1.48(1.06-2.04)$ & $1.55(1.14-2.20)$ & $1.53(1.17-2.87)$ & 0.001 \\
\hline $\mathrm{HsCRP}, \mathrm{mg} / \mathrm{dL}$ & $1.46(0.73-2.98)$ & $0.83(0.49-1.39)$ & $1.38(0.75-2.43)$ & $3.20(1.65-7.58)$ & $<0.001$ \\
\hline LVEF (\%) & $64.56 \pm 7.29$ & $65.13 \pm 6.94$ & $64.86 \pm 6.87$ & $63.68 \pm 7.95$ & $<0.001$ \\
\hline \multicolumn{6}{|l|}{ Medications at discharge } \\
\hline Statins, n (\%) & $5106(97.5)$ & $1707(97.8)$ & $1699(97.3)$ & 1699 (97.4) & 0.538 \\
\hline Aspirin, n (\%) & $5163(98.6)$ & $1716(98.3)$ & 1721 (98.6) & $1724(98.8)$ & 0.524 \\
\hline$\beta$-blockers, \% & $4137(79.0)$ & $1365(78.2)$ & $1347(77.2)$ & $1422(81.5)$ & 0.004 \\
\hline ACEI/ARB, \% & $2602(49.7)$ & $813(46.6)$ & $860(49.3)$ & $928(53.2)$ & $<0.001$ \\
\hline CCB, n (\%) & $2115(40.4)$ & $689(39.5)$ & $738(42.3)$ & $685(39.3)$ & 0.134 \\
\hline
\end{tabular}

The data are presented as mean \pm standard deviation, median (interquartile range) or number (\%). $B M I$ body mass index, $C A D$ coronary artery disease, $H b A 1 C$ glycosylated hemoglobin, FBG fasting blood glucose, $T C$ total cholesterol $H D L-C$ high-density lipoprotein cholesterol, $L D L-C$ low density lipoprotein cholesterol, $T G$ triglyceride, $H s C R P$ high-sensitivity C-reactive protein, $L V E F$ left ventricular ejection fraction, $A C E I$ angiotensin-converting enzyme inhibitors, $A R B$ angiotensin receptor blockers, $C C B$ calcium channel blockers

Table 2 Linear regression analysis between glucose metabolism and fibrinogen in patients with stable coronary disease

\begin{tabular}{lllll}
\hline Variable & Adjusted $\mathbf{R}^{\mathbf{2}}$ & Standard $\boldsymbol{\beta}$ & SEM & $\boldsymbol{P}$ value \\
\hline $\begin{array}{lllll}\text { Overall } \\
\text { HbA1C, \% }\end{array}$ & 0.027 & 0.166 & 0.010 & $<0.001$ \\
FBG, mmol/L & 0.014 & 0.120 & 0.006 & $<0.001$ \\
DM & & & & \\
HbA1C, \% & 0.045 & 0.213 & 0.016 & $<0.001$ \\
FBG, mmol/L & 0.021 & 0.146 & 0.009 & $<0.001$ \\
Non-DM & & & & \\
HbA1C, \% & 0.018 & 0.135 & 0.022 & $<0.001$ \\
FBG, mmol/L & 0.004 & 0.065 & 0.013 & $<0.001$
\end{tabular}

$D M$ diabetes mellitus, $\mathrm{HbA} 1 \mathrm{c}$ Hemoglobin A1c, FBG fasting blood glucose, $\mathrm{Cl}$ confidence interval, SEM standard error of estimate

with MACEs (per SD change of Fib: HR: 1.11, 95\% CI 1.01-1.24, $P=0.034$, medium Fib: HR: 1.15 , 95\% CI 0.901.47, $P=0.274$, high Fib: HR: 1.34, 95\% CI 1.02-1.75,
$P=0.035)$. Moreover, Fib was associated with composite endpoints including cardiovascular mortality, nonfatal MI, and nonfatal stroke (per SD change of Fib: HR: 1.17, 95\% CI 1.02-1.34, $P=0.023$, medium Fib: HR: 1.24, 95\% CI 0.87-1.77, $P=0.243$, high Fib: HR: $1.67,95 \%$ CI $1.14-2.45, P=0.008$, Table 3 ) after adjustment for other variables.

\section{Glucose metabolism, Fib levels, and cardiovascular outcomes}

Over a median follow-up time of 3.3 years (2.8 to 5.1 years), the incidence rates of MACEs in Pre-DM (8.5\%) and DM (11.7\%) groups were higher than those in the NGR (6.6\%) group $(p<0.001)$. Univariate Cox regression models showed that a baseline DM had a 1.77-fold higher risk of MACEs compared with the NGR group (HR: 1.77, 95\% CI 1.36-2.31, $p<0.001)$. Additional adjustment for other variables only slightly attenuated this association. However, the presence of pre-DM did not elevate MACEs risk compared with the NGR group in any adjusted model $(P>0.05)$ (Fig. 3). Moreover, we 
further assessed the risk of MACEs stratified according to both glucose metabolism status and Fib levels (Table 4). High Fib did significantly elevate the risk of MACEs in pre-DM when compared with the reference group, with adjusted HR of 1.61 (95\% CI 1.01-2.59, $P<0.05)$. The risks of MACEs in DM with medium Fib or high Fib groups were even higher, up to 1.86-fold (95\% CI 1.14-3.05, $P<0.05)$ and 2.28-fold (95\% CI 1.42-3.66, $P<0.05)$, compared with NGR and low Fib group.

Kaplan-Meier analysis with the Log-rank test indicated that patients with high Fib had the lowest cumulative event-free survival rates (Fig. 4a). Participants who had DM were least likely to be free of events among the three groups $(P<0.001$ for all comparisons). Nevertheless, there was no difference in the rate of incident CVD outcomes between pre-DM and NGR groups $(P=0.091$, Fig. $4 \mathrm{~b})$. Furthermore, we have assessed the prognostic significance of combining glucose metabolism and Fib levels, pre-DM with medium Fib, pre-DM with high Fib, DM with medium Fib, and DM with high Fib groups had lower cumulative incidence of MACE during follow-up compared with the reference group (NGR and low Fib group, all $P<0.01$ respectively, Fig. 4c).

Finally, we evaluated the combined effect of Fib and glucose status on predicting the risk of MACE occurrence. C-statistic values increased from 0.598 (95\% CI 0.571-0.625) for original prediction models of traditional risk factors to 0.613 (95\% CI $0.586-0.640)$ for the combination of Fib and glucose status [ $\Delta \mathrm{C}$-statistic: 0.015 (0.001-0.026), $P=0.022$, Table 5].

\section{Discussion}

In this cohort study, we found that serum Fib level was significantly associated with glucose metabolism (including FBG and $\mathrm{HbA1c}$ ) in Chinese stable CAD patients undergoing coronary angiography with or without DM. Cox regression analysis revealed that Fib was independently associated with MACEs during a median followup of 3.3 years. Interestingly, DM but not pre-DM was a significant predictor of MACEs when stratified by glucose metabolism status. However, patients with pre-DM and high Fib $(>3.39 \mathrm{mg} / \mathrm{dL})$ did have a 1.66-fold elevated risk of MACEs than those with normal blood glucose and low Fib. Moreover, adding the combination of Fib and glucose status to the model significantly improved the risk prediction for MACEs in the overall population. Our result, for the first time, provides evidence that patients with high Fib and pre-DM are prone to have a worse clinical prognosis.

\section{Fib and coronary artery disease}

Fib is a central factor in the chronic inflammatory process of atherosclerosis. As an essential component in atherosclerotic plaques, Fib can cause migration, the proliferation of smooth muscle cells, and increasing levels of proinflammatory cytokines, including IL-6 and TNF- $\alpha$ [21]. It also alters the integrity of endothelial cell layer and vascular wall permeability through ICAM-1 and P-selection, lures leukocytes form blood to the vessel wall through ligand-receptor, induces accumulation and activation of platelets [22], therefore orchestrates a multicellular inflammatory cascade with chronically detrimental clinical manifestations to some extent [23]. Consequently, epidemiological studies have demonstrated that Fib was significantly associated with cardiovascular outcomes in patients with CAD. AtheroGene study enrolled 1806 stable CAD patients with a median followup time of 3.5 years and found that per SD change of Fib was associated with 1.27-fold increased risk of cardiovascular death and non-fatal myocardial infarction [24]. Similarly, Mahmud et al. [25] reported that Fib $\geq 280 \mathrm{mg} /$ $\mathrm{dl}$ was associated with a 2.65 -fold elevated risk of 1-year major cardiovascular events after PCI. Whereas, the EPIC-Norfolk study included 16,850 patients who had no known cardiovascular history and showed that serum Fib lost its significance as a predictor for the seventeenyear risk of cardiovascular mortality after adjusted for other inflammation markers [26]. Despite the conflicting results of Fib and cardiovascular outcomes, the Canakinumab Antiinflammatory Thrombosis Outcomes Study (CANTOS) [27] and Colchicine Cardiovascular Outcomes Trial (COLCOT) [28] did provide encouragement that antiinflammation treatment may be useful in coronary artery disease. Thus, more evidence for the value of Fib in cardiovascular risk stratification in different subgroups were needed.

\section{Fib, glucose metabolic disorder, and diabetic vascular complications}

Concerning the relationship between Fib and glucose metabolic disorders, it has been demonstrated that Fib functioned as a crucial modulator of hemostatic balance

\footnotetext{
(See figure on next page.)

Fig. 2 Linear regression analysis of the relationship between glucose metabolism and FIB. a Linear regression analysis of the relationship between glucose metabolism [HbA1c (a1), FBG (a2)] and FIB in overall participants with CAD. $\mathbf{b}$ Linear regression analysis of the relationship between glucose metabolism [HbA1c (b1), FBG (b2)] and FIB in CAD patients with DM. c Linear regression analysis of the relationship between glucose metabolism [HbA1c (c1), FBG (c2)] and FIB in CAD patients without DM. FIB fibrinogen, DM diabetes mellitus, HbA1C HaemoglobinA1C, FBG fasting blood glucose
} 

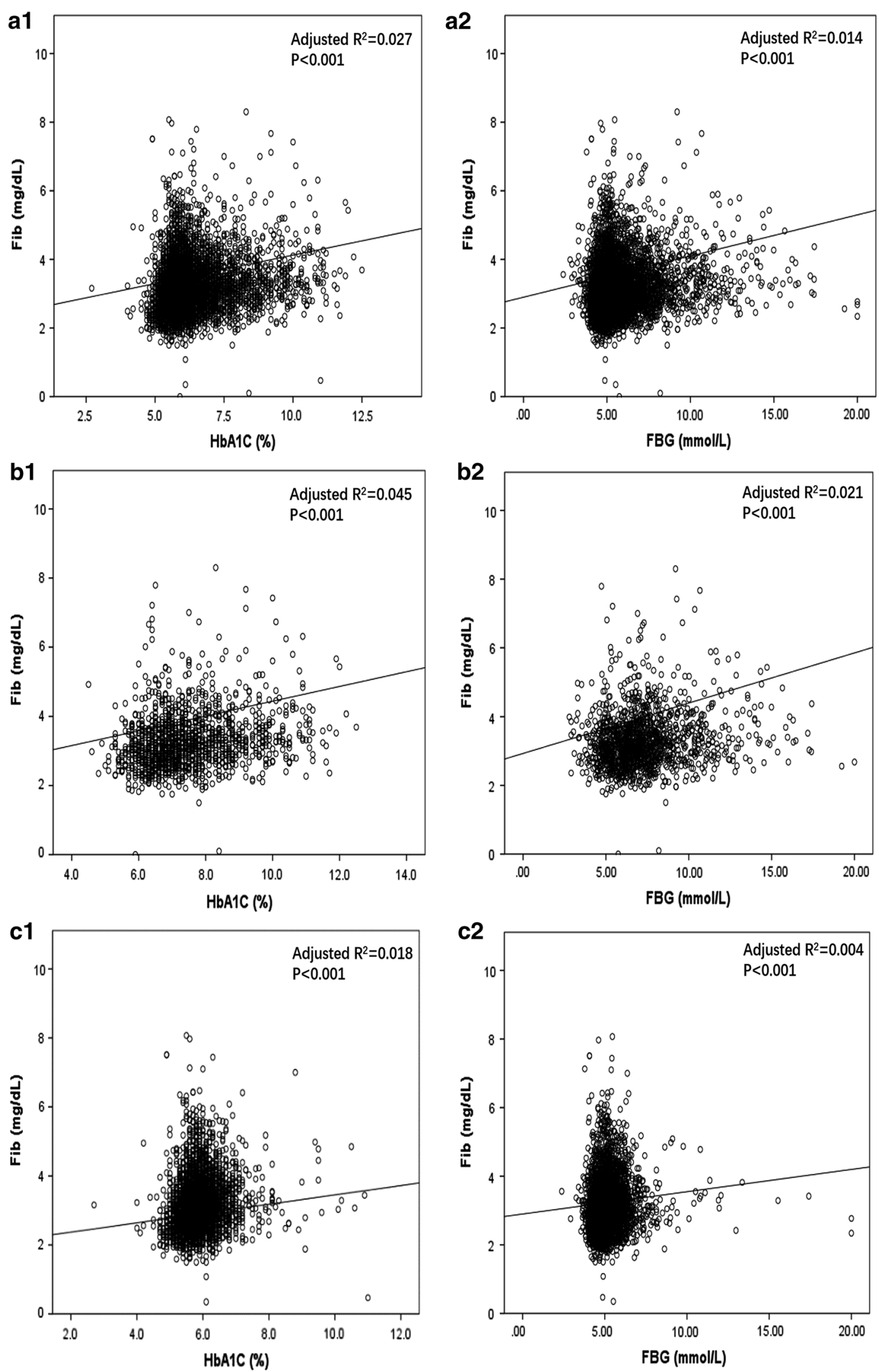
Table 3 Relation of the fibrinogen level and cardiovascular outcomes in univariate and multivariate survival analysis

\begin{tabular}{|c|c|c|c|c|}
\hline \multirow[t]{2}{*}{ Variables } & \multicolumn{2}{|c|}{ Univariate Cox regression } & \multicolumn{2}{|c|}{ Multivariate Cox regression } \\
\hline & Hazard ratio $(95 \% \mathrm{Cl})$ & $P$ value & Hazard ratio $(95 \% \mathrm{Cl})$ & $P$ value \\
\hline \multicolumn{5}{|l|}{ MACE } \\
\hline Fib (per SD change) & $1.18(1.09-1.27)$ & $<0.001$ & $1.11(1.01-1.24)$ & 0.034 \\
\hline Low Fib & Reference & - & Reference & - \\
\hline Medium Fib & $1.30(1.03-1.64)$ & 0.029 & $1.15(0.90-1.47)$ & 0.274 \\
\hline High Fib & $1.57(1.26-1.97)$ & $<0.001$ & $1.34(1.02-1.75)$ & 0.035 \\
\hline \multicolumn{5}{|l|}{ Composite endpoints } \\
\hline Fib (per SD change) & $1.26(1.13-1.40)$ & $<0.001$ & $1.17(1.02-1.34)$ & 0.023 \\
\hline Low Fib & Reference & - & Reference & - \\
\hline Medium Fib & $1.49(1.06-2.10)$ & 0.022 & $1.24(0.87-1.77)$ & 0.243 \\
\hline High Fib & $2.05(1.49-2.84)$ & $<0.001$ & $1.67(1.14-2.45)$ & 0.008 \\
\hline
\end{tabular}

Fib fibrinogen. Model adjusted for age, sex, body mass index, smoking, hypertension, family history of coronary artery disease, left ventricular ejection fraction, low density lipoprotein cholesterol, high lipoprotein cholesterol, Ln-transformed triglyceride, Ln-transformed high-sensitivity C-reactive protein, and creatinine

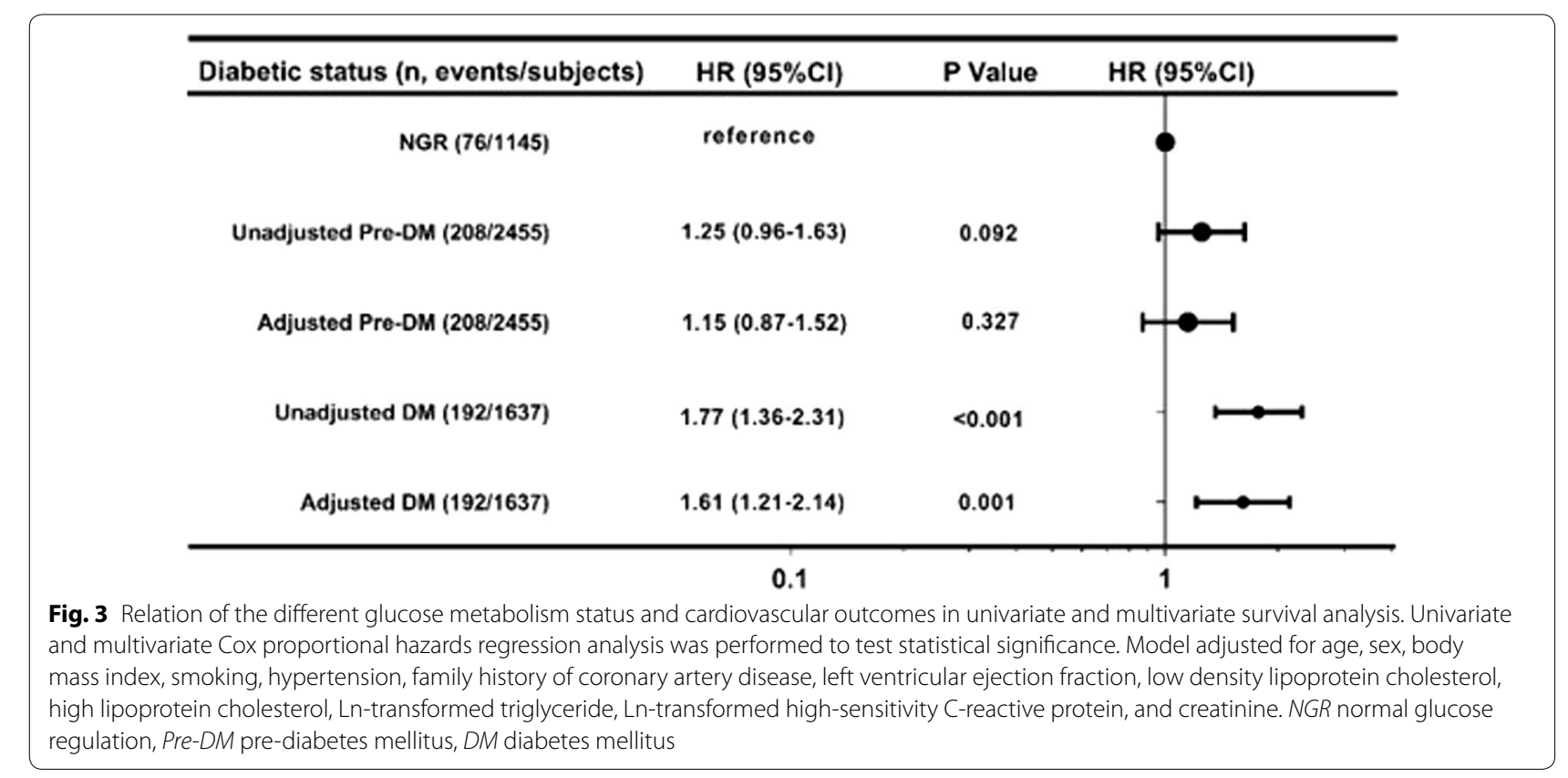

and inflammatory processes in diabetes [22, 29]. Impaired glucose metabolism and the status of insulin resistance result in oxidative stress, inflammatory response, coagulation activation, and accelerated atherosclerosis pathogenesis [30]. Fib synthesis was significantly promoted in pre-DM and type 2 diabetes, $[17,31]$ especially in those with high levels of polyhedral erythrocytes [32]. HbA1c was reported to have a positive effect on Fib level in DM [33]. Zhang et al. [15] found that Fib was associated with FBG and HbA1c in patients with ACS and DM, but not in those without DM. However, it must be emphasized that the result should be appraised with caution due to the small sample size (103 DM and 308 non-DM). On the contrary, our results demonstrated that Fib was significantly associated with FBG and HbA1c in 5237, newonset CAD patients either with or without DM (1637 $\mathrm{DM}$ and 3600 non-DM). Another reason for the conflicting result may be the difference in the study population, ACS versus stable CAD.

Furthermore, what is actually more important is whether Fib exerts an independent role in augmenting the risk of diabetic vascular complications. Fib independently associated with long-term microvascular disorders of type 2 DM, including diabetic nephropathy [34] and diabetic retinopathy (DR) [17], which increased the risk of cardiovascular events [35]. Interestingly, the Veterans 
Table 4 Fibrinogen levels in relation to cardiovascular events in patients with different glucose metabolism status

\begin{tabular}{|c|c|c|c|}
\hline & \multirow{2}{*}{$\begin{array}{l}\text { Events/subjects } \\
476 / 5237\end{array}$} & \multicolumn{2}{|l|}{ HR (95\% Cl) } \\
\hline & & Crude model & Adjusted model \\
\hline \multicolumn{4}{|l|}{ NGR } \\
\hline Low Fib & $25 / 510$ & Reference & Reference \\
\hline Medium Fib & $28 / 365$ & $1.56(0.91-2.67)$ & $1.47(0.85-2.52)$ \\
\hline High Fib & $23 / 270$ & $1.81(1.03-3.18)^{*}$ & $1.56(0.87-2.80)$ \\
\hline \multicolumn{4}{|l|}{ Pre-DM } \\
\hline Low Fib & $55 / 791$ & $1.36(0.85-2.19)$ & $1.24(0.78-2.00)$ \\
\hline Medium Fib & $72 / 814$ & $1.81(1.15-2.85)^{*}$ & $1.58(0.99-2.51)$ \\
\hline High Fib & $81 / 850$ & $1.95(1.25-3.05)^{*}$ & $1.61(1.01-2.59) *$ \\
\hline \multicolumn{4}{|l|}{ DM } \\
\hline Low Fib & $45 / 445$ & $2.09(1.28-3.41)^{*}$ & $1.81(1.13-2.92) *$ \\
\hline Medium Fib & $60 / 567$ & $2.15(1.35-3.43)^{*}$ & $1.86(1.14-3.05)$ * \\
\hline High Fib & $87 / 625$ & $2.89(1.85-4.50)^{*}$ & $2.28(1.42-3.66) *$ \\
\hline
\end{tabular}

Model adjusted for age, sex, body mass index, smoking, hypertension, family history of coronary artery disease, left ventricular ejection fraction, low density lipoprotein cholesterol, high lipoprotein cholesterol, Ln-transformed triglyceride, Ln-transformed high-sensitivity C-reactive protein, and creatinine. * p-value $<0.05$. Fib fibrinogen, NGR normal glucose regulation, Pre-DM prediabetes mellitus, $D M$ diabetes mellitus

Affairs Diabetes Trial [17] found that intensive glycemic control was associated with a decrease in the progression of DR in DM participants with lower Fib levels but not in DM patients with higher Fib levels, which suggests that there exists an interaction between glycemic control and Fib level.

Fib and cardiovascular outcomes in patients with coronary artery disease and different glucose metabolism status Glucose metabolism abnormalities are usual in patients with stable CAD and associated with a worse prognosis. Patients with CAD but without apparent glucose abnormalities were recommended to evaluate their glycemic state [36]. Prediabetes was associated with a worse prognosis in individuals with stable CAD only when combined with other cardiometabolic disorders [37-39]. Several previous studies demonstrated that the measurement of Fib provided incremental value to risk assessment in CAD patients with DM. The Gargano Heart Study has shown that Fib was an independent predictor of adverse major cardiovascular outcomes, including non-fatal myocardial infarction, stroke, and cardiovascular deaths, in
320 CAD patients with DM during an average follow-up time of 64.2 months [40]. That relationship was then validated in 1466, angiographic-proven stable CAD patients with DM over an average follow-up of 20.2 months [41] and 308 ACS patients with DM during a median followup of 27.5 months [15]. The Casale Monferrato Study demonstrated that Fib significantly associated with both 11-year all-cause and cardiovascular mortality in type 2 diabetes [42]. However, ADVANCE Study performed a nested case-cohort study in 2865 patients with DM and cardiovascular diseases and showed that IL-6 levels but not Fib levels added predictive value to diabetic macrovascular events and mortality [43]. The conflicting results between ADVANCE study and our study may due to its study population (including participants with stable CAD or MI). As the European Society of Cardiology emphasized recently, the dynamic nature of the CAD progression leads to different clinical presentations, which can have long, stable periods but can also become unstable at any time, typically attributed to an acute atherothrombotic event due to plaque rupture or erosion [44]. Thus, Fib levels may play various roles in ACS and chronic coronary syndromes (CCS). Besides, the predictive ability of Fib even changed over time in the DM population with ACS [15]. Nonetheless, there was no study available to evaluate the association between Fib levels and cardiovascular events in pre-DM status currently. To our best knowledge, this is the first study to investigate the combined effect of Fib and glucose metabolic status on MACEs risk. We found that patients with DM and medium Fib or high Fib had 1.86-fold and 2.28-fold increased risk of MACEs during the follow-up. Moreover, pre-DM alone was not an independent predictor of cardiovascular risk, but high Fib and pre-DM did have a worse cardiovascular prognosis, suggesting subclinical inflammation affects the prognosis of participants with impaired glucose metabolism.

The present study had several limitations. Firstly, since this is a single-center observational cohort study, some unmeasured confounders from the non-random assignment of exposure might influence the results of this study. Secondly, longer follow-up duration and larger sample population in this study will be required to investigate the prognostic value of pre-DM alone or combination with Fib in the long-term outcomes. Thirdly, this study only enrolled participants with stable CAD, which indicates that our results may only be applicable to stable CAD patients.

(See figure on next page.)

Fig. 4 Kaplan-Meier analysis according to different fibrinogen levels (a), glucose metabolism status (b), and status of both fibrinogen levels and glucose metabolism (c) 

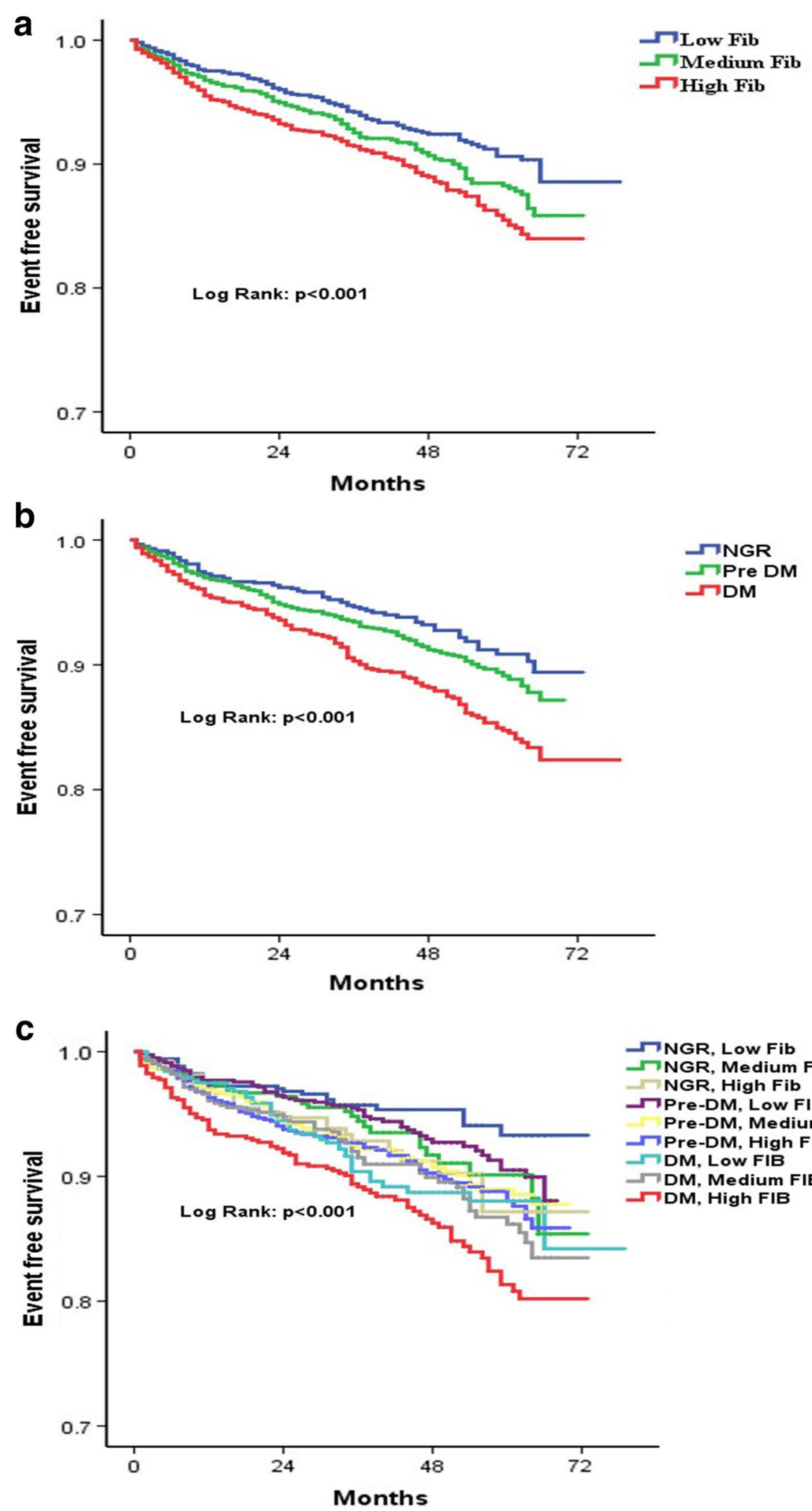


\section{Table 5 Incremental predictive values of glucose metabolism status and fibrinogen levels for cardiovascular outcomes}

\begin{tabular}{llll}
\hline & $\begin{array}{l}\text { C-statistic (95\% } \\
\text { Cl) }\end{array}$ & $\begin{array}{l}\Delta \text { C-statistic (95\% } \\
\text { CI) }\end{array}$ & p-value \\
\hline $\begin{array}{llll}\text { Original model } \\
\begin{array}{l}\text { Original } \\
\text { model + GF }\end{array}\end{array}$ & $0.598(0.571-0.625)$ & - & - \\
\hline
\end{tabular}

C-statistic and $\Delta C$-statistic were used to interpret efficiency of the models and the incremental value of adding fibrinogen levels into original model. Original model included age, sex, body mass index, smoking, hypertension, family history of coronary artery disease, left ventricular ejection fraction, low density lipoprotein cholesterol, high lipoprotein cholesterol, Ln-transformed triglyceride, Ln-transformed high-sensitivity C-reactive protein, and creatinine. Original Model indicates the C-statistic for MACEs (cardiovascular mortality, non-fatal myocardial infarction, stroke and post-discharge unplanned revascularization)

\section{Conclusions}

Fib levels were associated with FBG and HbA1c and could be used as an independent predictor of MACEs in stable CAD patients. Patients with pre-DM and high Fib but not pre-DM alone had a worse prognosis. Furthermore, patients with DM and high Fib had the highest risk of MACEs. Fib may provide incremental value in the cardiovascular risk stratification of pre-DM and DM patients.

\begin{abstract}
Abbreviations
CAD: Coronary artery disease; DM: Diabetes mellitus; Pre-DM: Prediabetes mellitus; NGR: Normal glucose regulation; FBG: Fasting blood glucose; HbA1c: Glycosylated hemoglobinA1c; ACS: Acute coronary syndromes; Fib: Fibrinogen; MI: Myocardial infarction; PCl: Percutaneous coronary artery intervention; CABG: Coronary artery bypass grafting; MACEs: Major adverse cardiovascular events; BMI: Body mass index; LVEF: Left ventricle ejection fraction; LDL-C: Low density lipoprotein cholesterol; HDL-C: High density lipoprotein cholesterol; TG: Triglyceride; HsCRP: High-sensitivity C-reactive protein; DR: Diabetic retinopathy.
\end{abstract}

\section{Acknowledgements}

We thanked patient advisers for the information they provided.

\section{Authors' contributions}

JJ L, NQ W planned the study, Y G, YL G, SL L, HW S conducted a survey, SL L analyzed the data and wrote the article. NQ W contributed to the drafting. All authors read and approved the final manuscript.

\section{Funding}

This work was partially supported by the Capital Health Development Fund (201614035) and CAMS Major Collaborative Innovation Project (2016-I2M-1011) awarded to J.-J.L.

The study sponsors did not participate in the study design; the collection, analysis, or interpretation of data; the writing of the manuscript; or the decision to submit the manuscript for publication.

\section{Availability of data and materials}

The datasets used and/or analysed during the current study are available from the corresponding author on reasonable request.

\section{Ethics approval and consent to participate}

The study complied with the principles of the Declaration of Helsinki and was approved by the ethical review board of Fuwai Hospital (Beijing, China). Written informed consent was obtained from all participants.

\section{Consent for publication}

Not applicable.

\section{Competing interests}

The authors declare that they have no competing interests.

Received: 1 February 2020 Accepted: 9 March 2020

Published online: 19 March 2020

\section{References}

1. Sugimoto MA, Ribeiro ALC, Costa BRC, et al. Plasmin and plasminogen induce macrophage reprogramming and regulate key steps of inflammation resolution via annexin A1. Blood. 2017;129:2896-907.

2. Soma P, Pretorius E. Interplay between ultrastructural findings and atherothrombotic complications in type 2 diabetes mellitus. Cardiovasc Diabetol. 2015;14:96.

3. Willeit P, Thompson SG, Agewall S, et al. Inflammatory markers and extent and progression of early atherosclerosis: meta-analysis of individual-participant-data from 20 prospective studies of the PROG-IMT collaboration. Eur J Prev Cardiol. 2016;23:194-205.

4. McEvoy JW, Nasir K, DeFilippis AP, et al. Relationship of cigarette smoking with inflammation and subclinical vascular disease: the multi-ethnic study of atherosclerosis. Arterioscler Thromb Vasc Biol. 2015;35:1002-10.

5. Harshfield EL, Sims MC, Traylor M, et al. The role of haematological traits in risk of ischaemic stroke and its subtypes. Brain. 2020;143:210-21.

6. Papageorgiou N, Briasoulis A, Hatzis G, et al. Coronary artery atherosclerosis in hypertensive patients: the role of fibrinogen genetic variability. Rev Esp Cardiol (Engl Ed). 2017;70:34-41.

7. Guedes AF, Carvalho FA, Moreira C, et al. Essential arterial hypertension patients present higher cell adhesion forces, contributing to fibrinogendependent cardiovascular risk. Nanoscale. 2017;9:14897-906.

8. Ang L, Behnamfar O, Palakodeti S, et al. Elevated baseline serum fibrinogen: effect on 2-year major adverse cardiovascular events following percutaneous coronary intervention. J Am Heart Assoc. 2017;6(11):e006580.

9. Kaptoge S, Di Angelantonio E, Pennells L, et al. C-reactive protein, fibrinogen, and cardiovascular disease prediction. N Engl J Med. 2012;367:1310-20

10. Danaei G, Finucane MM, Lu Y, et al. National, regional, and global trends in fasting plasma glucose and diabetes prevalence since 1980: systematic analysis of health examination surveys and epidemiological studies with 370 country-years and 2.7 million participants. Lancet. 2011;378:31-40.

11. Wang $L, G a o P, Z$ Zhang $M$, et al. Prevalence and ethnic pattern of diabetes and prediabetes in China in 2013. JAMA. 2017;317:2515-23.

12. Donath MY, Shoelson SE. Type 2 diabetes as an inflammatory disease. Nat Rev Immunol. 2011;11:98-107.

13. Cruz NG, Sousa LP, Sousa MO, et al. The linkage between inflammation and type 2 diabetes mellitus. Diabetes Res Clin Pract. 2013;99:85-92.

14. Grossmann V, Schmitt VH, Zeller T, et al. Profile of the immune and inflammatory response in individuals with prediabetes and type 2 diabetes. Diabetes Care. 2015;38:1356-64.

15. Zhang L, Xu C, Liu J, et al. Baseline plasma fibrinogen is associated with haemoglobin A1c and 2-year major adverse cardiovascular events following percutaneous coronary intervention in patients with acute coronary syndrome: a single-centre, prospective cohort study. Cardiovasc Diabetol. 2019;18:52.

16. Baker NL, Hunt KJ, Stevens DR, et al. association between inflammatory markers and progression to kidney dysfunction: examining different assessment windows in patients with type 1 diabetes. Diabetes Care. 2018;41:128-35. 
17. Azad N, Agrawal L, Emanuele NV, et al. Association of PAl-1 and fibrinogen with diabetic retinopathy in the Veterans Affairs Diabetes Trial (VADT). Diabetes Care. 2014;37:501-6.

18. Ferreira-Gonzalez I, Busse JW, Heels-Ansdell D, et al. Problems with use of composite end points in cardiovascular trials: systematic review of randomised controlled trials. BMJ. 2007;334:786.

19. Diagnosis and classification of diabetes mellitus. Diabetes Care. 2011;34(Suppl 1):S62-9.

20. Chevret S, Seaman S, Resche-Rigon M. Multiple imputation: a mature approach to dealing with missing data. Intensive Care Med. 2015;41:348-50.

21. Lu PP, Liu JT, Liu N, et al. Pro-inflammatory effect of fibrinogen and FDP on vascular smooth muscle cells by IL-6, TNF-alpha and iNOS. Life Sci. 2011;88:839-45.

22. Pretorius E. Platelets as potent signaling entities in type 2 diabetes mellitus. Trends Endocrinol Metab. 2019;30:532-45.

23. Davalos D, Akassoglou K. Fibrinogen as a key regulator of inflammation in disease. Semin Immunopathol. 2012;34:43-62.

24. Sinning JM, Bickel C, Messow CM, et al. Impact of C-reactive protein and fibrinogen on cardiovascular prognosis in patients with stable angina pectoris: the AtheroGene study. Eur Heart J. 2006;27:2962-8.

25. Mahmud E, Behnamfar O, Lin F, et al. Elevated serum fibrinogen is associated with 12-month major adverse cardiovascular events following percutaneous coronary intervention. J Am Coll Cardiol. 2016:67:2556-7.

26. Ahmadi-Abhari S, Luben RN, Wareham NJ, et al. Seventeen year risk of all-cause and cause-specific mortality associated with C-reactive protein, fibrinogen and leukocyte count in men and women: the EPIC-Norfolk study. Eur J Epidemiol. 2013;28:541-50.

27. Ridker PM, Everett BM, Thuren T, et al. Antiinflammatory therapy with canakinumab for atherosclerotic disease. N Engl J Med. 2017;377:1119-31.

28. Tardif JC, Kouz S, Waters DD, et al. Efficacy and safety of low-dose colchicine after myocardial infarction. N Engl J Med. 2019;381:2497-505.

29. Pretorius $L$, Thomson GJA, Adams RCM, et al. Platelet activity and hypercoagulation in type 2 diabetes. Cardiovasc Diabetol. 2018;17:141.

30. Wittmann I, Nagy J. Are insulin resistance and atherosclerosis the consequences of oxidative stress? Diabetologia. 1996;39:1002-3.

31. Bardini G, Dicembrini I, Cresci B, et al. Inflammation markers and metabolic characteristics of subjects with 1-h plasma glucose levels. Diabetes Care. 2010:33:411-3.

32. Gajos G, Siniarski A, Natorska J, et al. Polyhedrocytes in blood clots of type 2 diabetic patients with high cardiovascular risk: association with glycemia, oxidative stress and platelet activation. Cardiovasc Diabetol. 2018;17:146.

33. Abdul Razak MK, Sultan AA. The importance of measurement of plasma fibrinogen level among patients with type- 2 diabetes mellitus. Diabetes Metab Syndr. 2019;13:1151-8.
34. Zhang J, Wang Y, Zhang R, et al. Serum fibrinogen predicts diabetic ESRD in patients with type 2 diabetes mellitus. Diabetes Res Clin Pract. 2018;141:1-9.

35. Garofolo M, Gualdani E, Giannarelli R, et al. Microvascular complications burden (nephropathy, retinopathy and peripheral polyneuropathy) affects risk of major vascular events and all-cause mortality in type 1 diabetes: a 10-year follow-up study. Cardiovasc Diabetol. 2019:18:159.

36. Grant PJ, Cosentino F. The 2019 ESC Guidelines on diabetes, pre-diabetes, and cardiovascular diseases developed in collaboration with the EASD: new features and the 'Ten Commandments' of the 2019 Guidelines are discussed by Professor Peter J. Grant and Professor Francesco Cosentino, the Task Force chairmen. Eur Heart J. 2019:40:3215-7.

37. Jin JL, Cao YX, Liu HH, et al. Impact of free fatty acids on prognosis in coronary artery disease patients under different glucose metabolism status. Cardiovasc Diabetol. 2019;18:134.

38. Jin JL, Cao YX, Zhang HW, et al. Lipoprotein(a) and cardiovascular outcomes in patients with coronary artery disease and prediabetes or diabetes. Diabetes Care. 2019;42:1312-8.

39. Vistisen D, Witte DR, Brunner EJ, et al. Risk of cardiovascular disease and death in individuals with prediabetes defined by different criteria: the Whitehall II study. Diabetes Care. 2018;41:899-906.

40. Pacilli A, De Cosmo S, Trischitta V, et al. Role of relationship between $\mathrm{HbA} 1 \mathrm{c}$, fibrinogen and $\mathrm{HDL}$-cholesterol on cardiovascular disease in patients with type 2 diabetes mellitus. Atherosclerosis. 2013;228:247-8.

41. Yang SH, Du Y, Zhang Y, et al. Serum fibrinogen and cardiovascular events in Chinese patients with type 2 diabetes and stable coronary artery disease: a prospective observational study. BMJ Open. 2017;7:e015041.

42. Bruno G, Merletti F, Biggeri A, et al. Fibrinogen and AER are major independent predictors of 11-year cardiovascular mortality in type 2 diabetes: the Casale Monferrato Study. Diabetologia. 2005;48:427-34.

43. Lowe G, Woodward M, Hillis G, et al. Circulating inflammatory markers and the risk of vascular complications and mortality in people with type 2 diabetes and cardiovascular disease or risk factors: the ADVANCE study. Diabetes. 2014;63:1115-23.

44. Knuuti J, Wijns W, Saraste A, et al. 2019 ESC Guidelines for the diagnosis and management of chronic coronary syndromes. Eur Heart J. 2020;41:407-77.

\section{Publisher's Note}

Springer Nature remains neutral with regard to jurisdictional claims in published maps and institutional affiliations.
Ready to submit your research? Choose BMC and benefit from:

- fast, convenient online submission

- thorough peer review by experienced researchers in your field

- rapid publication on acceptance

- support for research data, including large and complex data types

- gold Open Access which fosters wider collaboration and increased citations

- maximum visibility for your research: over $100 \mathrm{M}$ website views per year

At BMC, research is always in progress.

Learn more biomedcentral.com/submissions 